EPJ Web of Conferences 32, 01008 (2012)

DOI: $10.1051 /$ epjconf/20123201008

(C) Owned by the authors, published by EDP Sciences, 2012

\title{
A relativistic model of electron cyclotron current drive efficiency in tokamak plasmas
}

\author{
Y.M. Hu, ${ }^{1}$ Y.J. Hu ${ }^{1}$ and Y.R. Lin-Liu ${ }^{2}$ \\ ${ }^{1}$ Institute of Plasma Physics and Center for Magnetic Fusion Theory, Chinese Academy of Sciences, \\ Hefei, China \\ ${ }^{2}$ Department of Physics and Center for Mathematics and Theoretical Physics, National Central \\ University, Taiwan
}

\begin{abstract}
A fully relativistic model of electron cyclotron current drive (ECCD) efficiency based on the adjoint function techniques is considered. Numerical calculations of the current drive efficiency in a tokamak by using the variational approach are performed. A fully relativistic extension of the variational principle with the modified basis functions for the Spitzer function with momentum conservation in the electron-electron collision is described in general tokamak geometry. The model developed has generalized that of Marushchenko's (N.B . Marushchenko, et al. Fusion Sci. \& Tech., 2009 ), which is extended for arbitrary temperatures and covers exactly the asymptotic for $u \gg 1$ when $Z \rightarrow \infty$, and suitable for ray-tracing calculations.
\end{abstract}

\section{The dimensionless ECCD efficiency}

For tokamak plasmas, define $\Theta=T_{e} / m c^{2}, h=B / B_{\max }, \gamma=\sqrt{1+2 \Theta x^{2}}, x=u / u_{0}, u_{0}=c \sqrt{2 \Theta}$, $w=(\gamma-1) m c^{2}, \quad \tilde{\chi}=\chi_{0} v_{e 0} B_{\max } / u_{0}, \quad v_{e 0}=n e^{4} \ln \Lambda^{e / e} / 4 \pi m^{2} \varepsilon_{0}^{2} u_{0}^{3}, \quad f_{m}=n_{e} f_{m t} / \pi^{3 / 2} u_{0}^{3}, \quad f_{m t}=$ $\sqrt{\Theta \pi / 2} \exp (-\gamma / \Theta) / K_{2}\left(\Theta^{-1}\right)$. Here $T_{e}, m, c, w, u, n_{e}, e, \ln \Lambda^{e / e}, B, B_{\max }, \chi_{0}, K_{2}$ are, respectively, electron temperature, electron mass, light speed, electron kinetic energy, momentum per unit mass, electron density, electron elementary charge, the Coulomb logarithm, magnetic field, the maximum of $B$ on the flux surface, response function, the 2th-order modified Bessel function of the second kind. From the above definition, the dimensionless ECCD efficiency defined in Ref.[1,2] can be expressed as

$$
\zeta^{*}=\frac{n_{e}\left\langle j_{\|}\right\rangle}{2 \pi \varepsilon_{0}^{2} T_{e} Q}=\frac{4\langle h\rangle}{\ln \Lambda^{e / e}} \frac{\int \gamma^{2} d \gamma D_{l} f_{m t} \tilde{L} \tilde{\chi}}{\int \gamma^{2} d \gamma D_{l} f_{m t}},
$$

where $j_{\|}=-e B\left\langle\int d \Gamma \chi S_{r f}\left(f_{M}\right)\right\rangle$ is the wave driven current parallel to the local magnetic field B, $Q=-e B\left\langle\int d \Gamma w S_{r f}\left(f_{M}\right)\right\rangle$ is the absorbed wave power density, and $\langle\ldots\rangle, d \Gamma$ and $S_{r f}\left(f_{M}\right)$ are, respectively, the flux-surface average, volume element and rf-induced quasilinear diffusion operator in velocity space;[3] $D_{l}$ is a wave diffusion factor which is a function of wave field and plasma parameter, $\tilde{L} \equiv \frac{\partial}{\partial \gamma}+\frac{n_{\|}}{\sqrt{2 \Theta}} \frac{\partial}{\partial x_{\|}}$, where $n_{\|}$is the parallel index of refraction. For trapped electrons $\tilde{\chi}=0$. For passing electrons $\tilde{\chi}$ satisfies the following equation:

$$
\left\langle\frac{h}{|\xi|} C_{e} \tilde{\chi}\right\rangle=-\operatorname{sgn}\left(u_{\|}\right) \frac{x}{\gamma}
$$

where $\xi=\boldsymbol{u}_{\|} / \boldsymbol{u}, C_{e} \tilde{\chi}=C_{e}^{l}\left(\tilde{\chi} f_{m}\right) / f_{m}$, and $C_{e}^{l}$ is the linearized relativistic collision operator. Using the collisional model of Taguchi,[4] and introducing the adiabatic invariant $\lambda=u_{\perp}^{2} B_{\max } / u^{2} B$, we write the solution of Eq. (2) as $\tilde{\chi}=\operatorname{sgn}\left(u_{\|}\right) F(x) H(\lambda)$ with

Eq. (2) can be rewritten

$$
H(\lambda)=\frac{1}{2} \int_{\lambda}^{1} \frac{d \lambda^{\prime}}{<\left(1-\lambda^{\prime} h\right)^{\frac{1}{2}}>}
$$

$$
C_{e}^{1} F(x)+\left(1-\frac{1}{f_{c}}\right) v_{e}(x) F(x)=-\frac{1}{f_{c}} \frac{x}{\gamma}
$$

where $f_{c}=\frac{3}{2}\left\langle h^{2}\right\rangle \int_{0}^{1} H\left(\lambda^{\prime}\right) d \lambda^{\prime}, C_{e}^{1} F(x)$ is the first Legendre harmonic of the linearized relativistic collision operator, $[5,6]$ and $v_{e}(x)$ is the pitch angle scattering rate. 


\section{Hirshman's Variational principle}

To solve Eq.(4) by using Hirshman's Variational principle,[7] we use the following basis functions:

$$
F(x)=\sum_{i=1}^{4} c_{i} g_{i}, g_{i}=\frac{x^{i+1}}{\gamma^{\kappa}},
$$

where $\kappa=1$ is the basis function adopted in Ref. [8](bf1), and $\kappa=2$ is another basis function which will give the exact solution in the limit of $Z \rightarrow \infty(\mathrm{bf} 2)$.

In Fig.1, the response functions $F_{1}$ and $F_{2}$, respectively corresponding to the basis functions bf1 and bf2, determined from the variational principle are in good agreement with the exact numerical solutions of equation (4) when $x<4$.0. For $x>4$ some discrepancies are observed which may not be significant, as only the products of $F$ and $f_{m t}$ appear in the calculations for ECCD efficiency.
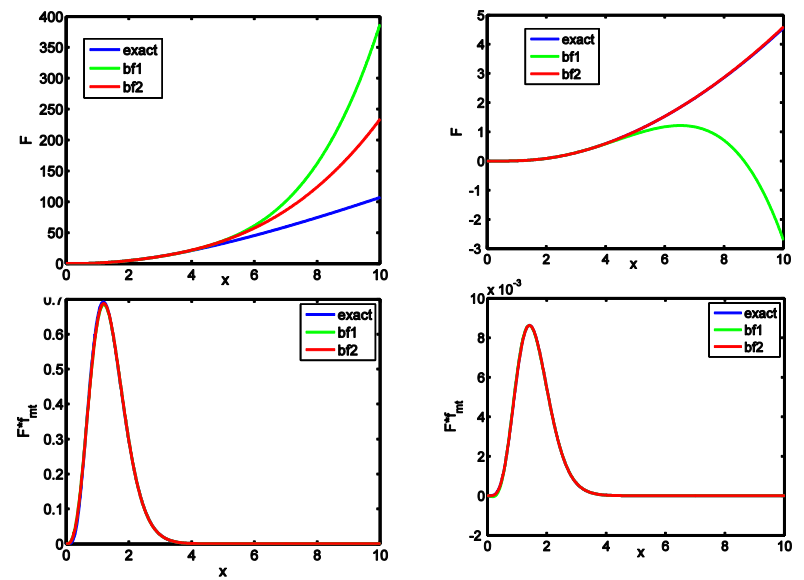

Fig. 1. Comparison of the response function $F$ (up) and $F * f_{m t}$ (down) with different $Z$ for $\Theta=0.1$, $f_{c}=0.5$. left: $Z=1$, right: $Z=100$.

\section{Conclusions}

A model of ECCD efficiency is developed based on the relativistic Coulomb collision operators. Taguchi's approximation is used to simplify the collision operators and Hirshman's variational principle to calculate the current drive response function, a new set of basis functions for the variational calculations, which gives the exact result in the Lorentz-Gas limit $(Z \rightarrow \infty)$, is proposed. The variational matrix elements are calculated without making the low-temperature expansion, and an accurate and computationally efficient ECCD model appears to be possible.

\section{Acknowledgments}

This work is supported by the National Nature Science Foundation of China Under Grant No. 10975156 and by Ministry of Science and Technology of China under contract No. 2009GB105001.

\section{References}

1. Y. R. Lin-Liu., V. S. Chan, and R. Prater, Phys. Plasmas, 10, 4064 (2003).

2. Y. M. Hu, Y. J. Hu, and Y. R. Lin-Liu, Fusion Sci. Technol. 59, 684 (2011).

3. C.F. Kennel and F. Engelmann, Phys. Fluids 9, 2377 (1966).

4. M. Taguchi, Plasma Phys. Control. Fusion, 31, 241 (1989).

5. S.T. Beliaev and G.I. Budker, Sov. Phys. Dokl. 1,218(1956).

6. B.J. Braams and C. F.F. Karney, Phys. Fluids B 1, 1355(1989).

7. S. P. Hirshman, Phys. Fluids, 23, 1238(1980).

8. N. B. Marushchenko, C. D. Beidler, and H. Maassberg, Fusion Sci. Technol. 55, 180 (2009). 